Provided for non-commercial research and education use. Not for reproduction, distribution or commercial use.

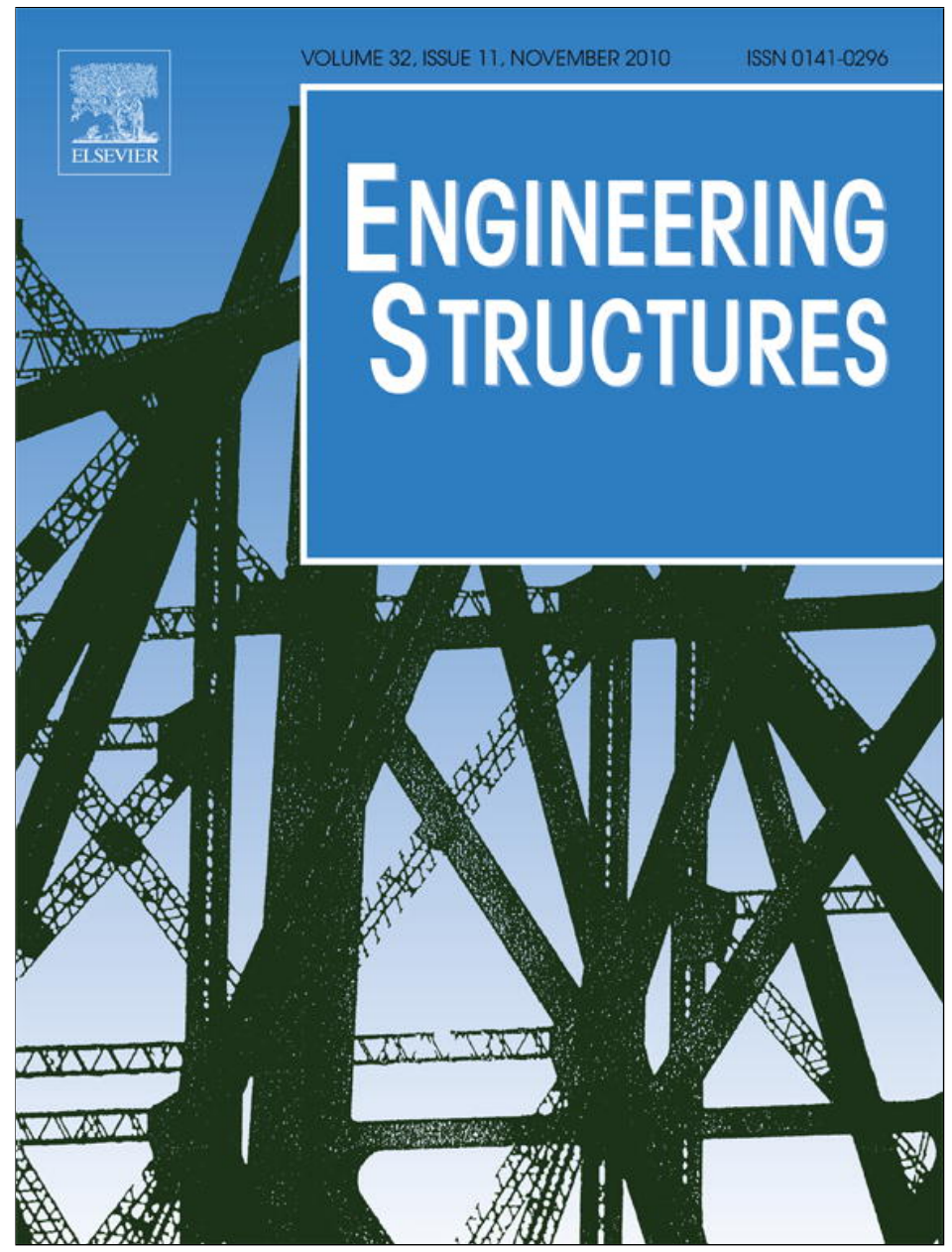

This article appeared in a journal published by Elsevier. The attached copy is furnished to the author for internal non-commercial research and education use, including for instruction at the authors institution and sharing with colleagues.

Other uses, including reproduction and distribution, or selling or licensing copies, or posting to personal, institutional or third party websites are prohibited.

In most cases authors are permitted to post their version of the article (e.g. in Word or Tex form) to their personal website or institutional repository. Authors requiring further information regarding Elsevier's archiving and manuscript policies are encouraged to visit:

http://www.elsevier.com/copyright 


\title{
Coupled and uncoupled nonlinear elastic finite element models for monotonically loaded sheathing-to-framing joints in timber based shear walls
}

\author{
Johan Vessby ${ }^{\mathrm{a}, \mathrm{b}, *}$, Erik Serrano $^{\mathrm{a}}$, Anders Olsson ${ }^{\mathrm{a}}$ \\ a School of Technology, Linnaeus University, Lückligs Plats 1, S-351 95 Växjö, Sweden \\ ${ }^{\mathrm{b}}$ Tyréns AB, Storgatan 40, S-352 31 Växjö, Sweden
}

\section{A R T I C L E I N F O}

\section{Article history:}

Received 4 January 2010

Received in revised form

7 April 2010

Accepted 31 May 2010

Available online 6 September 2010

\section{Keywords:}

Shear walls

Coupled model

Sheathing-to-framing joint

Finite element method

\begin{abstract}
A B S T R A C T
Four different elastic models for sheathing-to-framing connections are presented and evaluated on a single connection level and on a shear wall level. Since the models are elastic in their nature they are suitable mainly for cases where the sheathing-to-framing connections are subjected to monotonically increasing displacements. Of the four models one is uncoupled and the others are coupled with respect to the two perpendicular displacement directions in a two-dimensional model. Two of the coupled models are non-conservative, while the third is conservative, indicating a path independency with respect to the work done to reach a defined state of deformation. When the different models are compared it is obvious that the uncoupled model gives strength and stiffness values higher than the others; however it is not obvious which of the models to use in a shear wall analysis, each of the models having its advantages and disadvantages. For the experimental data used as input in the analyses of this study however, a coupled non-conservative model seems the most appropriate.
\end{abstract}

(c) 2010 Elsevier Ltd. All rights reserved.

\section{Introduction}

\subsection{Background}

In many timber structures shear walls are used to resist lateral loads such as wind loads. The wind load acts on an exterior wall or on the roof and is then transferred to horizontal diaphragms in the flooring or in the roof. These diaphragms in turn transfer the forces to the shear walls that stabilize the house. The walls are normally designed with timber members in the top rail, the bottom rail and in the studs. A sheathing material such as gypsum board, plywood, oriented strand board (OSB) or fibre board is fastened to one of the sides, or both sides, of this timber frame. The sheet is fastened to the timber frame by means of screws or nails along the perimeter of the sheet and along the centre stud. The structural components of a timber shear wall are shown in Fig. 1. Forces are transmitted in tension, compression and in shear to the bottom rail that interacts with the foundation through contact, friction and by means of connectors.

In order to predict the capacity of such shear walls various models have been suggested and utilized. Many of these models are designed to be implemented using the finite element method.

\footnotetext{
* Corresponding author at: School of Technology, Linnaeus University, Lückligs Plats 1, S-351 95 Växjö, Sweden. Tel.: +460470 708845.

E-mail address: johan.vessby@Inu.se (J. Vessby).
}

One crucial matter when formulating finite element models of timber structures in general and timber based shear walls in particular are the assumptions used for defining the behaviour of connectors, in this particular case the sheathing-to-framing joints that transfer the forces between the structural elements.

\subsection{Previous work}

It is a well established fact that the characteristics of the individual sheathing-to-framing joints in shear walls are crucial in the role of defining the strength and the stiffness of the wall, see for instance [1,2]. Due to their importance, also rather elaborate models have been developed, e.g. [3] including also the post-peak behaviour, [2] and hysteretic response, e.g. [4,5]. It is crucial that these models represent the behaviour of the physical connection well in order to build relevant three-dimensional structures. Attempts to build such three-dimensional models have been made by several researchers including e.g. [6,7].

Typically, connector elements are used to model the loaddisplacement properties of the sheathing-to-framing joint. Their properties may be nonlinear and the model may introduce (nonlinear) single spring elements or spring pair elements, see [8]. A standard 1-dimensional single spring model, i.e. with a defined force versus elongation relation, may be used to represent the load-displacement behaviour in any direction. One drawback with such a single spring model is that of zero stiffness for deformation perpendicular to the elongation of the spring. This may give 


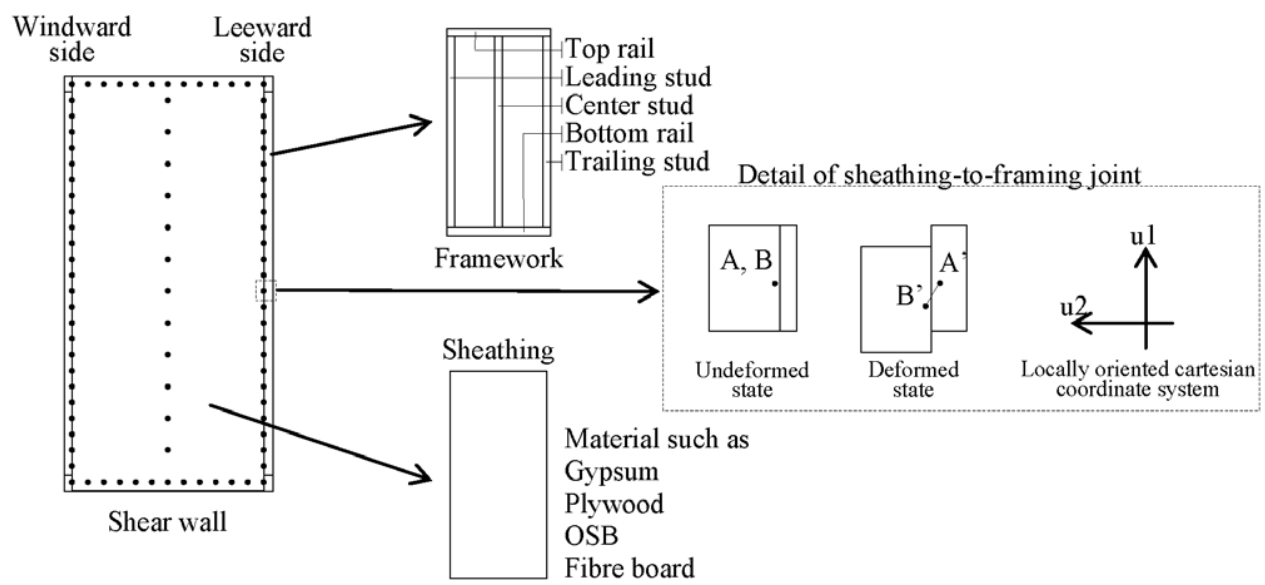

Fig. 1. Structural components of a timber shear wall. The fastener indicated (connecting point A with point B) is shown both in undeformed and deformed state.

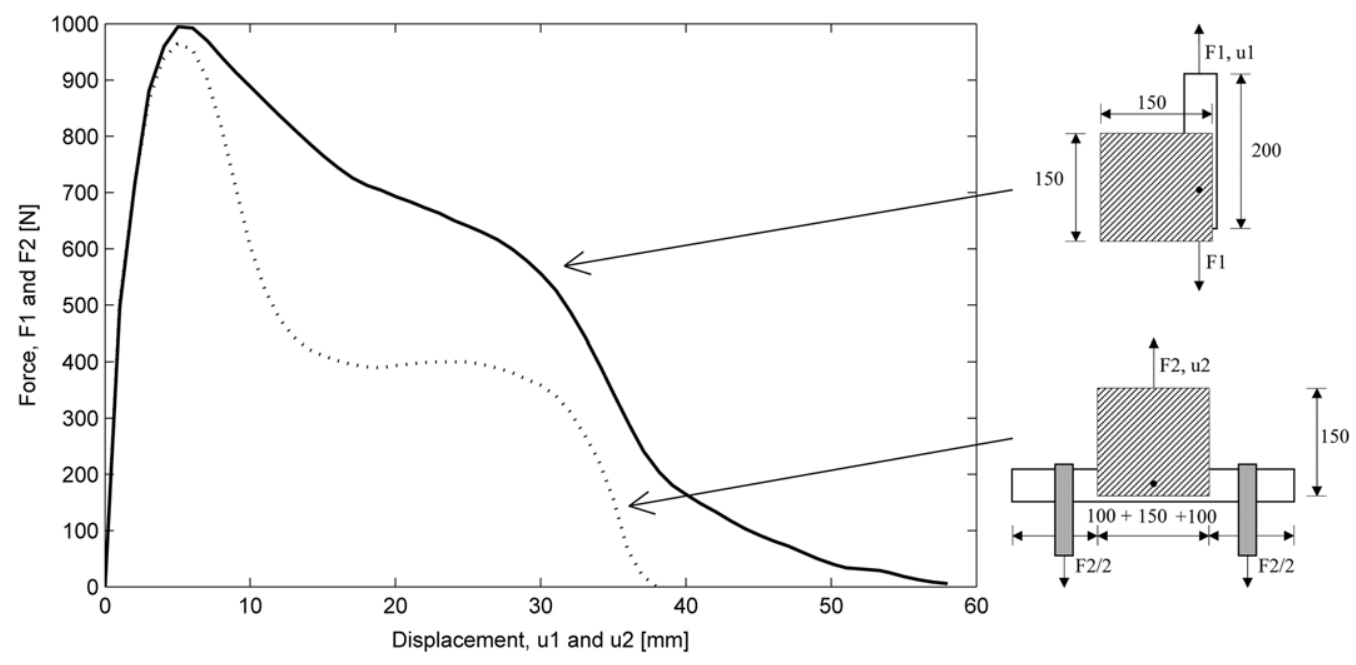

Fig. 2. Load-displacement relations for loading in parallel and in perpendicular directions respectively to the timber member.

numerical difficulties if the trajectory is dramatically changed. Another drawback is that the load-displacement characteristics only allow for one of the two local orthogonal directions ( $u 1$ and $u 2$ ) to be considered, see Fig. 1. A common approach to overcome these disadvantages is to use an uncoupled nonlinear model consisting of two orthogonally oriented springs, see $[9,10]$. In such a model each of the two springs may be given any load-displacement property. However this spring pair model typically overestimates the strength and stiffness of nonlinear sheathing-to-framing joints for any loading pattern involving a mixed mode loading path, i.e. a combination of displacement in the $u 1$ and $u 2$ directions. This may be compensated for by scaling the spacing between the joints in a shear wall to obtain balance with respect to the energy absorbed [9]. It may also be compensated for by modifying the load-displacement properties of the individual joint until they fit an experimentally obtained load-displacement relation for a larger structure, such as for instance a shear wall, [11]. Both these approaches may give a reasonable response to a specific load case on a shear wall, but in the first case the geometry has to be changed during the analysis, which is both non-physical and time-consuming, and in the latter case the joint properties cannot be verified by experiments.

Another modification of the spring pair model has been suggested by Judd and Fonseca [8]. They used an uncoupled spring pair model and oriented the model with respect to the initial displacement trajectory. By using such an approximation the uncoupled spring pair model will not overestimate the strength, as long as the displacement continuously takes place along that initially specified trajectory (i.e. a radial displacement trajectory in the $u 1-u 2$ space). This approximation is efficient as long as the joint does not diverge from that initially determined trajectory. Once this happens, the model may still give high strength values. It may also be difficult to handle different load-displacement characteristic in the two orthogonal directions effectively, i.e. longitudinal and perpendicular to the grain, in such a model. Xu and Dolan [5] used the approximation suggested by Judd and Fonseca [8] to model shear walls subjected for cyclic loading. They used the same hysteretic model in the spring parallel to the initial trajectory as well as the spring perpendicular to the initial trajectory. This might be a choice that is acceptable for cyclic loading, but in the case of successively increasing loading the orthotropic characteristics of the joint, indicated for instance by the experimental results shown in Fig. 2 , should be included in the analyses.

\subsection{Objectives of the present study}

In order to model shear walls with higher accuracy and to really capture the actual response, one of the crucial points is to develop more accurate sheathing-to-framing joint models and these should not be too demanding in terms of computational cost. The main objective of this paper is to investigate the general behaviour of nonlinear elastic spring pair models and thus gaining knowledge about the possibilities and limitations related to the 
use of these in numerical analyses. The models should have the possibilities to include the different behaviour in the local $u 1$ and $u 2$ directions. Furthermore, the models should be possible to use for any monotonic loading situation in a shear wall without having to change joint properties or geometric parameters depending on the shear wall being analysed.

Below, four different spring pair models, one uncoupled and three coupled, are described and compared. In all cases the load-displacement characteristics are different in the directions parallel and perpendicular to the grain in the timber member respectively. For simplicity all models are formulated as being nonlinear elastic, but include softening behaviour, i.e. the joint has load bearing capacity after peak load, but this capacity diminishes as displacement increases.

Being elastic, the models are candidates for use in situations dominated by monotonic loading. The shear wall analyses performed here are two dimensional analyses, aiming at capturing the in-plane shear action of the shear wall. For structures involving two perpendicular shear walls, the presented approaches can still be used for each of the shear walls using a proper orientation of each of the two-dimensional walls. It is thus assumed that the outof-plane action within each shear wall, due to transverse loading or due to second order effects can be disregarded.

\section{Behaviour and modelling of sheathing-to-framing joints}

\subsection{Physical behaviour of joints}

The load-displacement relation of a single fastener in a timber member depends on the direction of the loading. This phenomenon is mainly due to the different stiffness in the perpendicular directions of the highly orthotropic wood material. Experiments performed by Girhammar et al. [12] and evaluated by Vessby [13] shows the load-displacement characteristic in the two directions for an $8 \mathrm{~mm}$ thick hardboard (wet process fibre board, quality HB.HLA2) fastened to the timber member (Pine, C24, $45 \times 120 \mathrm{~mm}$ in cross-section). The fastener was an annular ringed shank nail (Duofast) with the dimensions $50 \times 2.1 \mathrm{~mm}$ and the yield capacity $f_{y} \approx 400-500 \mathrm{MPa}$. In the experiments the sheathingto-framing joints were loaded parallel and perpendicular to the timber member respectively. These local directions will be referred to with respect to load and displacement as $F 1$ and $u 1$ (parallel) and $F 2$ and $u 2$ (perpendicular) respectively. Mean value curves, based on 10 such experiments in each direction subjected to displacement controlled loading, are shown in Fig. 2. In the figure, the differences between the two directions are obvious. In the postpeak region the joint load is considerably higher in loading parallel to the timber member as compared to loading perpendicular to the timber member.

In the case of an orthotropic sheathing material such as OSB or plywood, four curves would be needed to define the behaviour in the two directions since the orientation of the sheathing (outer layers being oriented in one way or another) might differ in relation to the timber member. One pair of relations would then be used to model the one orientation of the sheet and the other pair to model the other orientation. Thus, also for the case of an orthotropic sheathing material, for any given fastener position only one pair of load-displacement curves is needed to describe the response of that specific fastener.

\subsection{Finite element modelling of joints}

In a two dimensional finite element model of a shear wall employed herein for comparison of joint models the timber
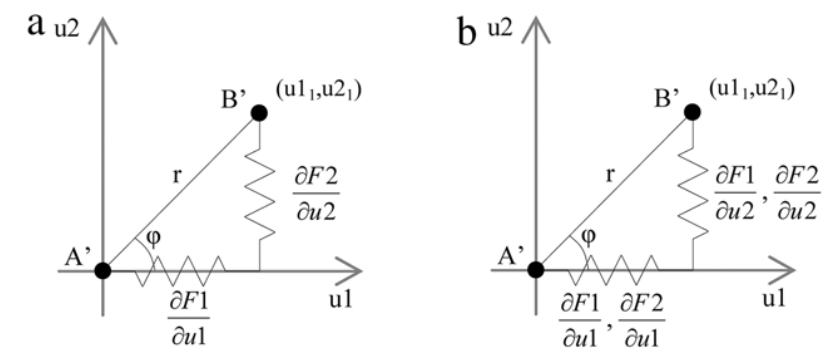

Fig. 3. Representation of the structural element used for modelling the sheathingto-framing joint in (a) an uncoupled manner and (b) in a coupled manner. The partial derivatives represent the tangential formulation to be used in the tangential stiffness matrix in the relation between the displacements $[u 1, u 2]$ and the forces $[F 1, F 2]$.

members are modelled as linear elastic beam elements with two translational and one rotational degree of freedom in each node. The sheathing is modelled using rectangular, linear elastic, plane stress elements. Since the timber members, as well as the sheathing members, are assumed to be linear elastic, all sources of material nonlinearity emanate from the sheathing-toframing joints or from the joints connecting the studs and rails. In the simulations the fibre orientation in the timber members is assumed to coincide with the direction of the member itself. Each sheathing-to-framing joint is modelled using a spring pair element, which adds stiffness in the two perpendicular directions, the one parallel to the timber member, referred to as $u 1$, and the one perpendicular to the timber member, referred to as $u 2$. The definitions used for an uncoupled and a coupled model are given in Fig. 3, showing a local coordinate system and two nodes $A^{\prime}$ and $B^{\prime}$. Node $A^{\prime}$ is located at the origin and node $B^{\prime}$ at the arbitrary point $\left(u 1_{1}, u 2_{1}\right)$. The current elongation of the spring pair element is denoted $r$, and is calculated from the two components of displacement $u 1_{1}$ and $u 2_{1}$.

One decisive choice that has to be made for a joint model is whether or not it should possess coupled behaviour. The joint model presented in Fig. 3(a) does not have such coupled behaviour. This implies that the stiffness in each direction does only depend upon the displacement in that direction such that $F 1=F 1(u 1)$ and $F 2=F 2(u 2)$. In such a case the tangential stiffness matrix $\mathbf{K}^{\tan }$ for the joint is formulated as

$\mathbf{K}^{\tan }=\left[\begin{array}{cc}\frac{\partial F 1}{\partial u 1} & 0 \\ 0 & \frac{\partial F 2}{\partial u 2}\end{array}\right]$

and it will contain nonzero elements only in the diagonal. If instead, as indicated in Fig. 3(b), the joint is given properties corresponding to a coupled behaviour such that $F 1=F 1(u 1, u 2)$ and $F 2=F 2(u 1, u 2)$, the tangential stiffness matrix $\mathbf{K}^{\mathrm{tan}}$ will be a full matrix (2) in the form of a Jacobian matrix.

$\mathbf{K}^{\tan }=\frac{\partial(F 1, F 2)}{\partial(u 1, u 2)}=\left[\begin{array}{ll}\frac{\partial F 1}{\partial u 1} & \frac{\partial F 1}{\partial u 2} \\ \frac{\partial F 2}{\partial u 1} & \frac{\partial F 2}{\partial u 2}\end{array}\right]$

$\mathbf{K}^{\mathrm{tan}}$ can be a symmetric matrix but in general, $\mathbf{K}^{\mathrm{tan}}$ is unsymmetrical. In addition, each of the terms of the matrix will vary in each increment of the analysis due to the nonlinearity of the functions $F 1$ and $F 2$.

Below four different definitions of the functions $F 1$ and $F 2$ are presented: one corresponds to an uncoupled model, and three to different coupled models. The functions $F 1$ and $F 2$ are based on experimental curves, i.e. the load versus displacement relation recorded in the two orthogonal directions, see Fig. 2. 
a

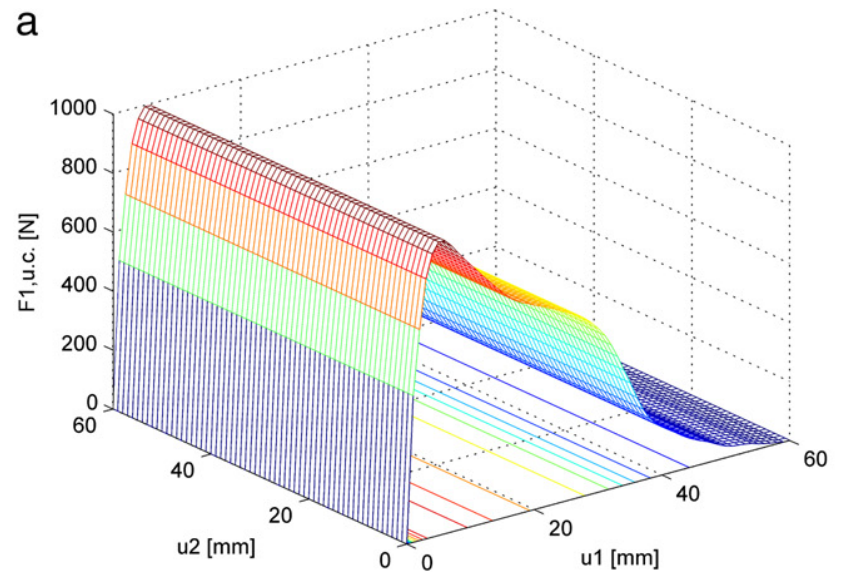

b

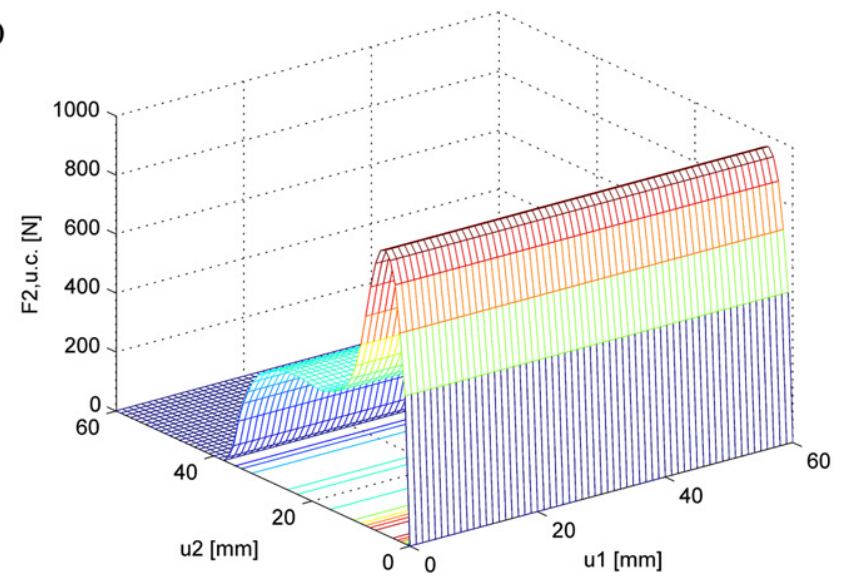

Fig. 4. The surfaces represent the forces (a) $F 1$ and (b) $F 2$ respectively if uncoupled behaviour is assumed.

Two of the coupled models described below give unsymmetrical tangential stiffness matrices, $\mathbf{K}^{\mathrm{tan}}$. This, in turn, means that the work done to reach a defined state of deformation depends on the path followed to reach that state, a behaviour which in continuum mechanics is commonly referred to as a Cauchy elastic (non-conservative) relation. Although the work done is path dependent, the force in the fastener for a given state of displacement is uniquely defined by the functions $F 1$ and $F 2$.

Taking into account that the failure process of the sheathingto-framing joint includes phenomena such as plasticity of the fastener, plastic deformation of the orthotropic wood and sheathing, and possibly also fracture of the wood and the sheathing, it is likely that the true behaviour should be path dependent, and this possibly both in terms of work and force (e.g. due to unloading). The Cauchy elastic approach chosen here has, however, some obvious advantages in terms of its simple formulation, and is a reasonable approximation if the joint is exposed to monotonic loading. That the work done can be path dependent can be concluded already by inspection of the uniaxial responses, cf. Fig. 2. If it can be shown that the work to failure is the same, or as an approximation does not differ considerably for the two principal directions, it could be beneficial to introduce a non-linear hyper-elastic formulation. This special case of Cauchy elasticity results in a full tangential stiffness matrix that is symmetric and thus the vector field defining the properties of the joint is conservative. In terms of engineering calculations, such a formulation is of considerable interest since the principle of force superposition would be valid, greatly facilitating design based on hand calculations. The third of the coupled models presented herein will possess such properties.

\section{Spring pair models}

\subsection{General remarks}

The two curves representing uniaxial loading in the two orthogonal directions, $F 1(u 1)$ and $F 2(u 2)$ respectively, are assumed known from experiments. In mixed mode loading of any joint an assumption has then to be done regarding the shape of the 3Dsurfaces representing the mixed mode behaviour for a general mode of loading, i.e. the functions $F 1=F 1(u 1, u 2)$ and $F 2=$ $F 2(u 1, u 2)$ respectively. One uncoupled model and three coupled models will be discussed here. Each of these will later be implemented in a finite element model of a shear wall.

\subsection{Uncoupled spring pair model}

In Fig. 4 the two surfaces representing the force in the direction parallel to the timber member, $F 1$, and the force in the direction perpendicular to the member, $F 2$, are shown. The fact that the model is uncoupled (abbreviated u.c.) implies that $F 1$ does not vary with respect to $u 2$ and $F 2$ does not vary with respect to $u 1$. This in turn leads to the fact that the isolines of the surfaces $F 1(u 1, u 2)$ and $F 2(u 1, u 2)$ are parallel with the $u 2$ - and $u 1$-axis respectively. Thus the partial derivatives, $\partial F 1 / \partial u 2$ and $\partial F 2 / \partial u 1$, are:

$$
\frac{\partial F 1}{\partial u 2}=0
$$

and

$$
\frac{\partial F 2}{\partial u 1}=0
$$

The tangential stiffness matrix will thus be a diagonal matrix:

$K_{\text {u.c. }}^{\mathrm{tan}}=\left[\begin{array}{cc}\frac{\partial F 1}{\partial u 1} & 0 \\ 0 & \frac{\partial F 2}{\partial u 2}\end{array}\right]$

and for full calibration of this model, only results from experiments in the two orthogonal directions are needed.

This uncoupled model implies that e.g. pulling the fastener parallel to the $u 1$-axis gives the same load-displacement response whether $u 2=0$ or anything else. This is of course not realistic since some kind of interaction, or rather coupling, between the two directions of loading certainly occurs in practice. Due to this, a coupled model is more realistic, and will therefore be the main candidate.

\subsection{Coupled model with rectangular outer bound}

In the two orthogonal directions it is assumed that the uniaxial force-displacement responses possess the properties $F 1(u 1) \geq 0$ and $F 2(u 2) \geq 0$ when $u 1 \leq u 1_{\max }$ and $u 2 \leq u 2_{\max }$ respectively and that $F 1(u 1)=0$ and $F 2(u 2)=0$ when $u 1>u 1_{\max }$ and $u 2>u 2_{\max }$ respectively. No force can thus be transmitted after certain displacements in each direction. In accordance with test results displayed in Fig. $2 u 1_{\max }$ and $u 2_{\max }$ are here set to $59 \mathrm{~mm}$ and $38 \mathrm{~mm}$ respectively.

To introduce the coupling, we define a 2-dimensional space where the $F 1$ and $F 2$ responses respectively depend on both $u 1$ and $u 2$. As no force can be transmitted if $u 1>u 1_{\max }$ or $u 2>u 2_{\max }$ the force-displacement response is non-zero over a rectangular area in the $u 1-u 2$-space defined by the values $u 1_{\max }$ and $u 2_{\max }$, obtained from uniaxial tests. A smooth surface for the coupled response can 

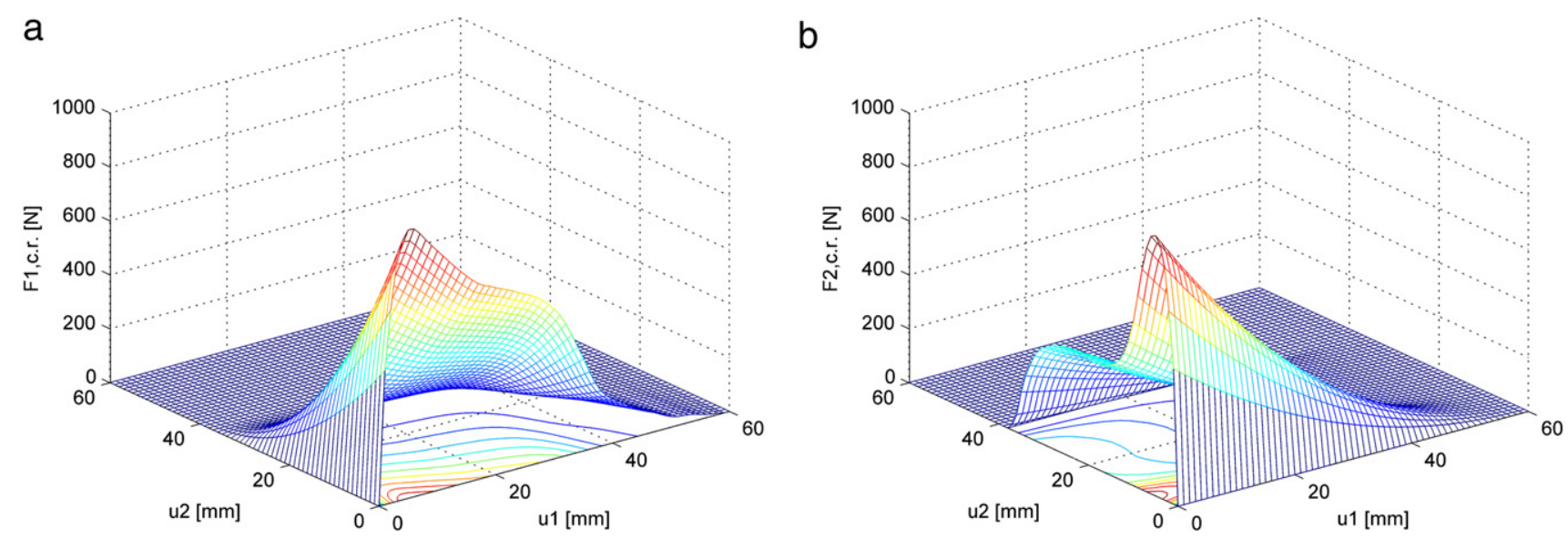

Fig. 5. The surfaces represent the forces (a) $F 1$ and (b) $F 2$ respectively assuming a quadratic decrease towards the outer bound.

then be obtained by choosing e.g.

$F 1(u 1, u 2)=F 1(u 1,0) \cdot\left(1-\frac{u 2}{u 2_{\max }}\right)^{\beta 1}$
$F 2(u 1, u 2)=F 2(0, u 2) \cdot\left(1-\frac{u 1}{u 1_{\max }}\right)^{\beta 2}$.

The parameters $\beta 1$ and $\beta 2$ are then chosen so as to get a good fit to experimental results. Of course, if experimental data is lacking, a straightforward choice would be $\beta 1=\beta 2=\beta$. The value of $\beta$ determines the strength of the coupling, $\beta=0$ generating the uncoupled model of the above, $\beta=1$ introducing a linear decrease of the uniaxial force response as a function of the displacement in the perpendicular direction, $\beta=2$ a quadratic decrease etc.. If $\beta=$ 1 constant values of the mixed derivatives of the Jacobian matrix (see (2)) are obtained, and the coupling terms in the tangential stiffness matrix $\mathbf{K}^{\mathrm{tan}}$ are constant. If instead $\beta=2$, the coupling terms will not be constant and the decrease of the surface will be quadratic. In this study, $\beta=2$ for the purpose of illustrating the influence of a rather strong coupling. This sheathing-to-framing model will be referred to as coupled-rectangular-outer bounds (abbreviated c.r.).

The two surfaces $F 1$ and $F 2$ obtained in this way are shown in Fig. 5(a) and (b) respectively. As mentioned above, the surfaces are defined as being nonzero within a rectangular area of the $u 1-u 2$-space. There is one important consequence of this. Defining the forces $F 1$ and $F 2$ as being nonzero within this subspace has one troublesome consequence. In uniaxial loading the deformation capacities (deformation at which the load reaches zero) are approximately 60 and $40 \mathrm{~mm}$ for the $u 1$ and $u 2$ directions respectively (Fig. 5$)$. For any radial load path $(u 1 / u 2=$ constant) the deformation capacity is however always larger than $40 \mathrm{~mm}$, and for some cases even larger than $60 \mathrm{~mm}$. This is probably unphysical. In order to consider this, an alternative concept regarding the coupling is presented below.

\subsection{Coupled model with smooth outer bound}

An alternative to the coupled model above would be to define a different interaction subspace. One such choice is to define the following subspace:

$$
\left(\frac{u 1}{u 1_{\max }}\right)^{m}+\left(\frac{u 2}{u 2_{\max }}\right)^{n}=1
$$

where $u 1_{\max }$ and $u 2_{\max }$ represent the above mentioned zero-force displacements in the two perpendicular directions. The choice of

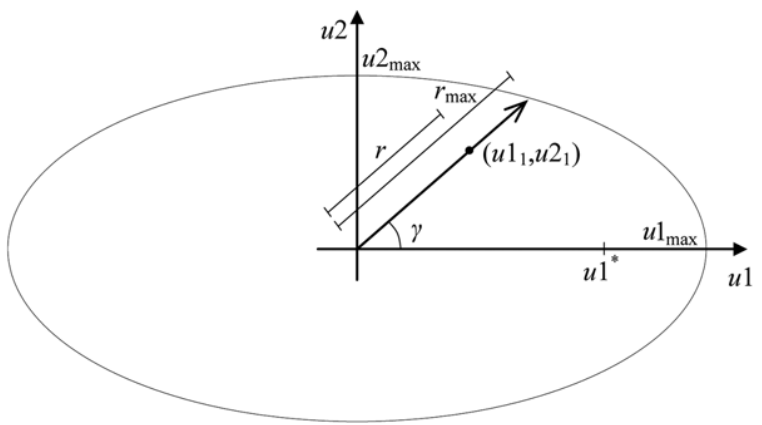

Fig. 6. Definitions of the parameters used for a coupled joint model with elliptic outer bound.

the parameters $m$ and $n$ in (7) should be based on experimental evidence. Here, for simplicity, $m=n=2$, resulting in the interaction being defined over an elliptic subspace. Note that letting $m=n=$ 1 will result in a linear relation and that $m=n=\infty$ will result in a rectangular outer bound, similar to the one above. Within the subspace (7), the force in any point is obtained by mapping the uniaxial curves $F 1(u 1,0)$ or $F 2(0, u 2)$ respectively to the line defined by the origin $(0,0)$ and the current point in deformation space, see Fig. 6. As an example, for the point $\left(u 1_{1}, u 2_{1}\right)$ an equivalent displacement $u 1^{*}$ is calculated to find the equivalent force $F 1\left(u 1^{*}, 0\right)$ :

$\frac{r}{r_{\max }}=\frac{u 1^{*}}{u 1_{\max }}$

where $r$ is the length from the origin to the current point $\left(u 1_{1}, u 2_{1}\right)$ and $r_{\max }$ is the length from the origin to the outer bound defined by (7) (ellipse for $m=n=2$ ) through the current point, cf. Fig. 6. Using the displacement $u 1^{*}$, the corresponding force $F 1\left(u 1^{*}, 0\right)$ is scaled for the current angle $\gamma_{1}$ with a scaling function. One such possible scaling function is:

$F 1\left(u 1_{1}, u 2_{1}\right)=\cos (\gamma) \cdot F 1\left(u 1^{*}, 0\right)$

where the angle $\gamma$ is defined by $\gamma=\tan ^{-1}\left(u 2_{1} / u 1_{1}\right)$.

The same principle is used for the force $F 2$ and with the above mentioned choice of $m=n=2$, the functions are nonzero within an elliptic subspace, see Fig. 7. This connector model will be referred to as coupled-elliptic-outer bounds (abbreviated c.e.).

\subsection{Coupled model with hyper-elastic properties}

By definition, a hyper-elastic model (abbreviated h.e.) means that the same amount of energy is used independent of how the current state of displacement in a single joint was reached. This 
a

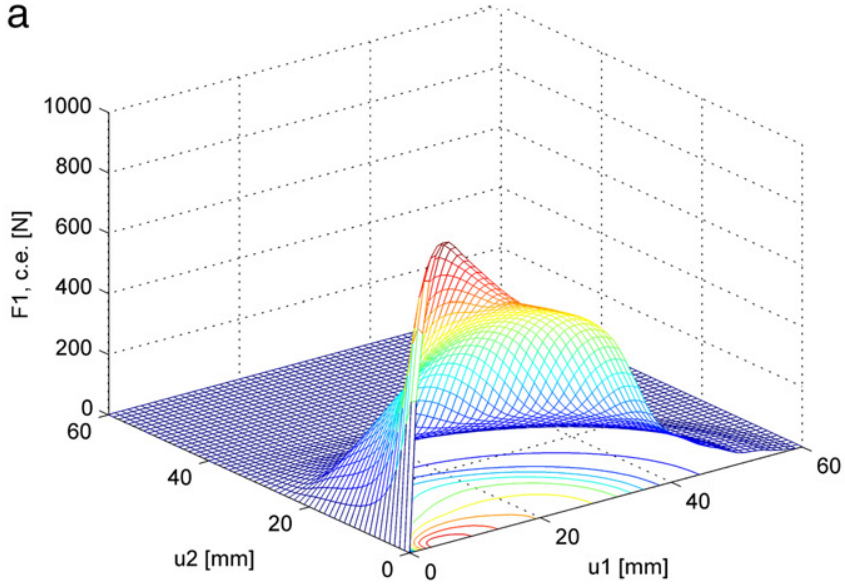

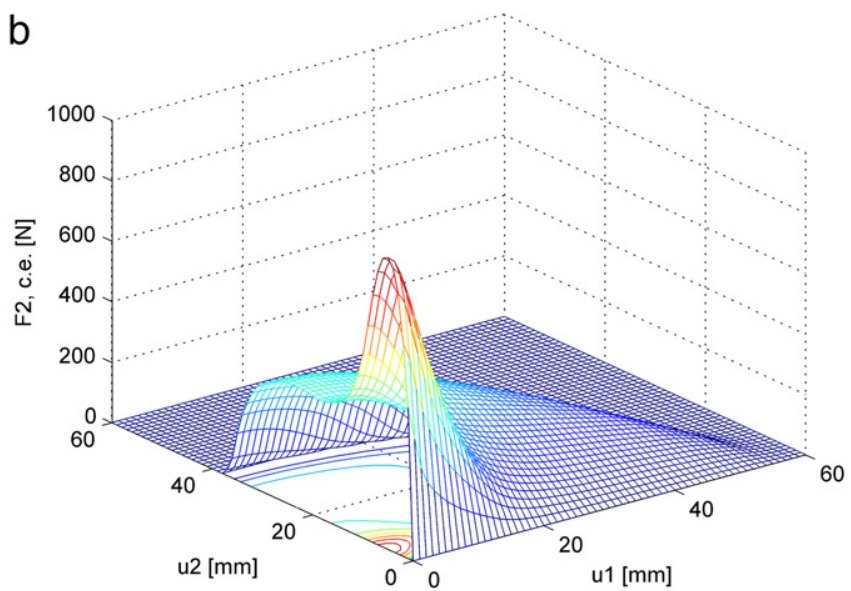

Fig. 7. The surfaces represent the forces (a) $F 1$ and (b) $F 2$ respectively assuming an elliptic outer bound.
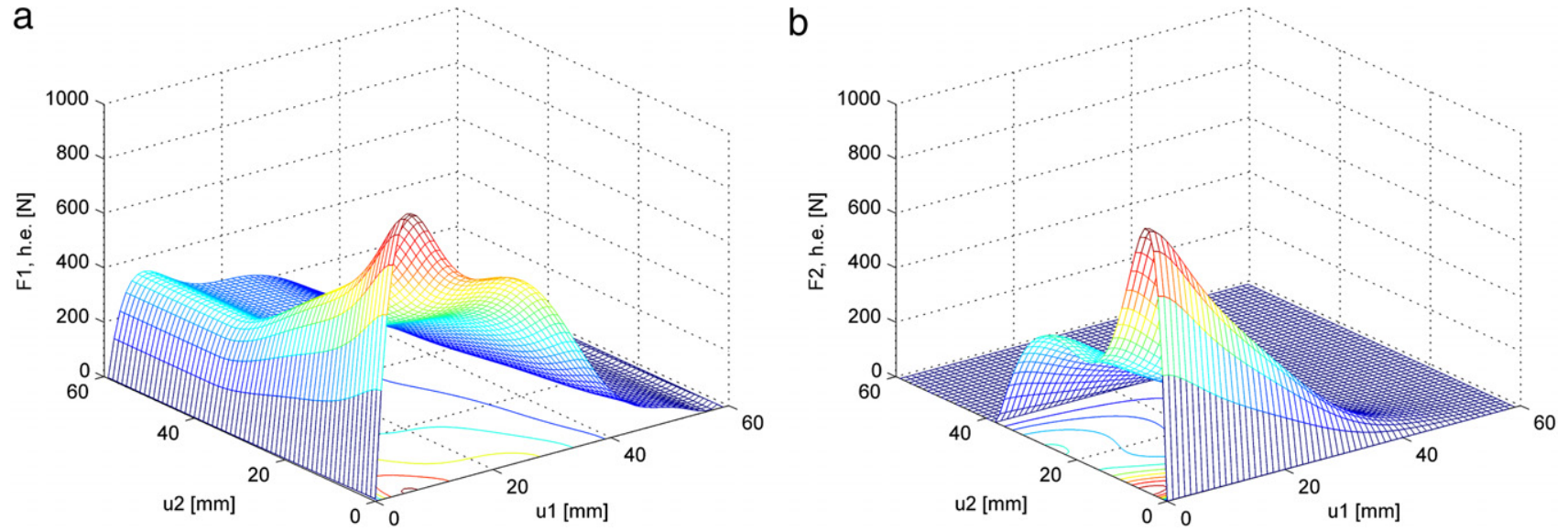

Fig. 8. The surfaces represent the forces (a) $F 1$ and (b) $F 2$ respectively assuming a conservative field.

corresponds to a conservative vector field that, per definition, has the properties of providing us with a symmetric tangential stiffness matrix since a prerequisite for a conservative field is that

$\frac{\partial F 1}{\partial u 2}=\frac{\partial F 2}{\partial u 1}$.

This in turn indicates that the uncoupled model first described is a special case of a hyper-elastic model since the two partial derivatives, i.e. the off-diagonal terms in $\mathbf{K}^{\mathrm{tan}}$, were both zero in that model.

In order to formulate in a general way a hyper-elastic model one can start with defining the two vector fields, each dependent on the two coordinates $u 1$ and $u 2$ as

$F 1(u 1, u 2)=\alpha(u 1) \cdot \varphi_{u 2}(u 2)$

$F 2(u 1, u 2)=\beta(u 2) \cdot \varphi_{u 1}(u 1)$

where the $\varphi$-functions represent the coupling between the two directions. The uniaxial responses are captured correctly if $\varphi_{u 1}(0)=\varphi_{u 2}(0)=1$ and the $\alpha$ and $\beta$-functions are equal to the uniaxial responses $F 1(u 1,0)$ and $F 2(0, u 2)$ respectively. Since we know that in a conservative field (10) should hold, we know that the $\varphi$-functions must fulfil

$\varphi_{u 2}(u 2)=B(u 2) \cdot c_{1}+c_{2}$

$\varphi_{u 1}(u 1)=A(u 1) \cdot c_{1}+c_{3}$

where the functions $A$ and $B$ are primitive functions of $\alpha$ and $\beta$ respectively, and the constants $c_{1}, c_{2}$ and $c_{3}$ are to be chosen. Since $A$ and $B$ are primitive functions of $\alpha$ and $\beta$ respectively we have that $A(u 1=0)=B(u 2=0)=0$. Therefore, in order to fulfil $\varphi_{u 1}(0)=\varphi_{u 2}(0)=1$ we must chose $c_{2}=c_{3}=1$. The trivial choice $c_{1}=0$ would then result in the uncoupled model previously described. Another way of choosing the constant $c_{1}$ would be so that one of the functions, e.g. $F 2$, declines to 0 along $u 1=u 1_{\text {max }}$. Since the choice here has to be made whether $F 1$ should be zero along $u 2=u 2_{\max }$ or $F 2$ should be zero along $u 1=u 1_{\max }$, the obvious choice is that $c_{1}$ is chosen so that the non-zero parts of the surface at the outer bound always has positive values.

From the experimental results in the present case the work to failure is different in the two principal directions, see Fig. 2. Fitting this experimental data to a hyper-elastic model and choosing $c_{1}$ such that $F 2=0$ for all $u 1>u 1_{\max }$ will result in the function derivative $\partial F 1 / \partial u 2$ being constant for $u 2>u 2_{\max }$, cf. Fig. 8, where the two fields $F 1$ and $F 2$ respectively are shown. The size of $F 1$ for $u 2>u 2_{\max }$ corresponds to the difference in work between $\int_{u 1=0}^{u 1_{\max }} F 1(u 1,0) \mathrm{d} u 1$ and $\int_{u 2=0}^{u 2_{\max }} F 2(0, u 2) \mathrm{d} u 2$.

\subsection{Total force response-vector sums}

One way to compare the models is to perform a finite element analysis using the different models and compare the results. This will be done later. Another method is to calculate and compare the resulting force for any state of displacement, i.e. the vector sum (v.s.) of the force components. This is done in accordance with (13) and the four comparable surfaces obtained are shown in Fig. 9.

$$
F_{v . s .}\left(u 1_{i}, u 2_{j}\right)=\sqrt{F 1\left(u 1_{i}, u 2_{j}\right)^{2}+F 2\left(u 1_{i}, u 2_{j}\right)^{2}} .
$$



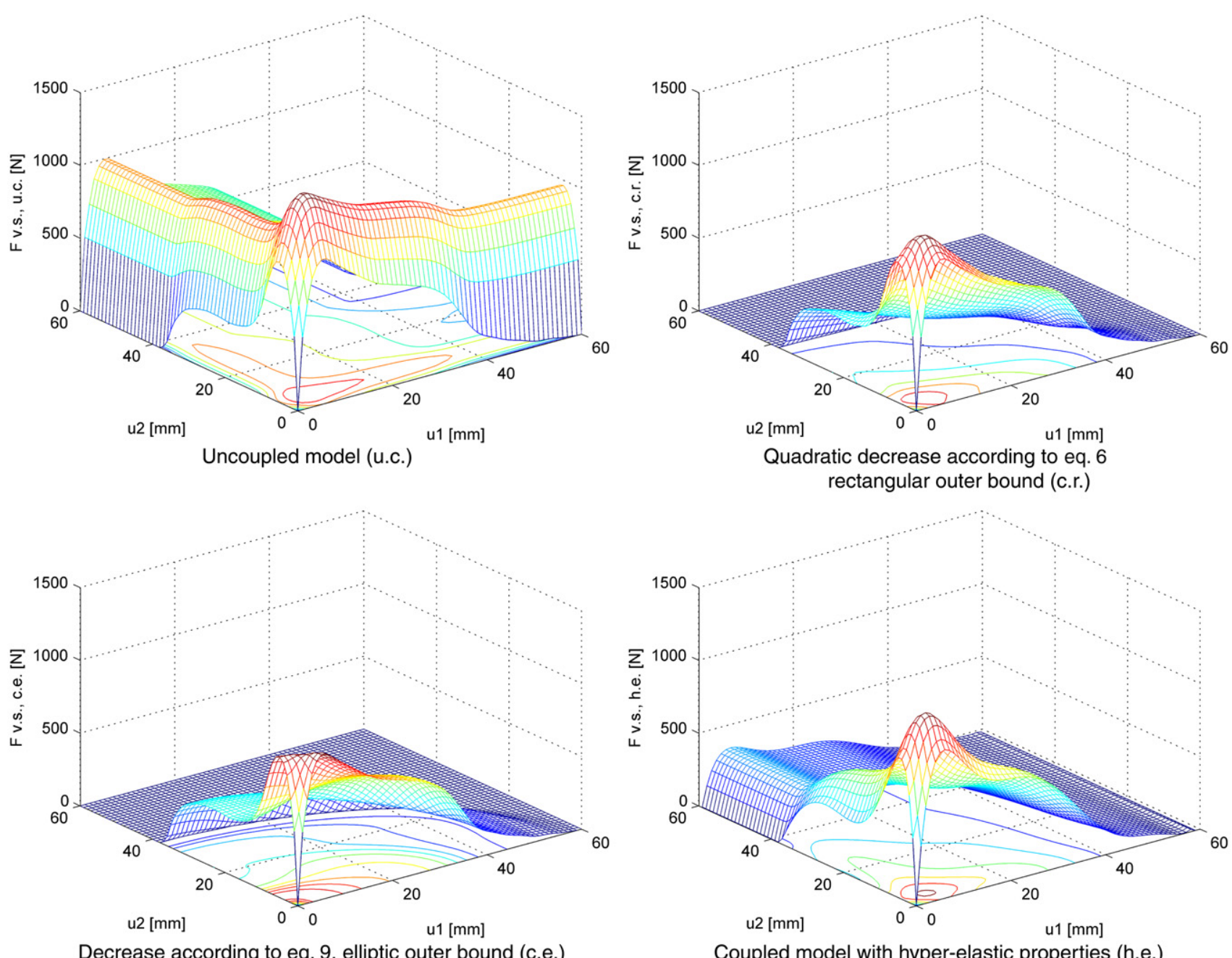

Fig. 9. Vector sum of the four sheathing-to-framing joint models respectively.

By looking at the surfaces at least three different observations can be made. The first is that for the uncoupled model, the vector sum is much larger than for the other models. The second observation that can be made is that there seems to be only a slight difference between the two non-conservative coupled models. The clearest difference between the two surfaces can be seen in the vicinity of $(u 1, u 2)=(2,2)$. In addition, even though not very clearly visible, the rectangular outer bound model is troublesome. It seems unrealistic that if the displacement is applied in the direction towards $\left(u 1_{\max }, u 2_{\max }\right)$, the forces in the joint will remain for longer elongation than if loaded in any other direction. This implies that the coupled-elliptic-outer bound model (cf. Eq. (8)) in this respect is the more realistic one of the non-conservative models.

The last of the three observations concerns the hyper-elastic model. This model is attractive in the sense that it is path independent, but for the current experimental data it results in a non-physical force response for large values of $u 2$ when $u 1$ is close to zero, as mentioned above.

\section{Simulation results}

\subsection{Analysis of a single joint}

From the results shown in Fig. 9 it is clear that the models discussed behave differently when implemented and used as input to a finite element analysis. As an example, it can be seen that if the joint is subjected to a translation in the direction $\varphi=45^{\circ}$, the vector sum is quite different depending which model is used. In order to show the differences a finite element model of a single joint with input from the four models described above was set up. One node is fixed to the origin and the other node is translated along a trajectory defined by four points at the coordinates $(5,1)$, $(-2,10),(-15,-10)$ and $(50,-50)$, see Fig. 10 . The trajectory is defined with the purpose of showing the differences in the models. The models are compared by plotting the vector sum of the forces $F 1$ and $F 2$ along the accumulated total length of the trajectory as previously suggested. The plot is shown in Fig. 10.

Three different observations are done. The first is that the uncoupled model behaves differently compared to the other models. Forces up to almost $1400 \mathrm{~N}$ are obtained at given points along the trajectory. This is not realistic since the experimentally obtained capacity in any of the two directions never exceeds 1000 $\mathrm{N}$, and for any in-between direction the capacity is not expected to be higher. Thus the model should be handled with caution. To a less extent this is also the case for the hyper-elastic model, although it does not overestimate the strength to the same extent as the uncoupled model does. The second observation is that the difference between the two other coupled models is limited. Slightly higher forces (vector sum) are obtained in the model with quadratic decrease, but the shapes of the curves are similar. The third observation addresses the issue that the models are all elastic. Since this is the case, the force response will always follow the predefined surfaces in Figs. 4, 5, 7 or 8 respectively, and unloading would result in a non-physical response. 


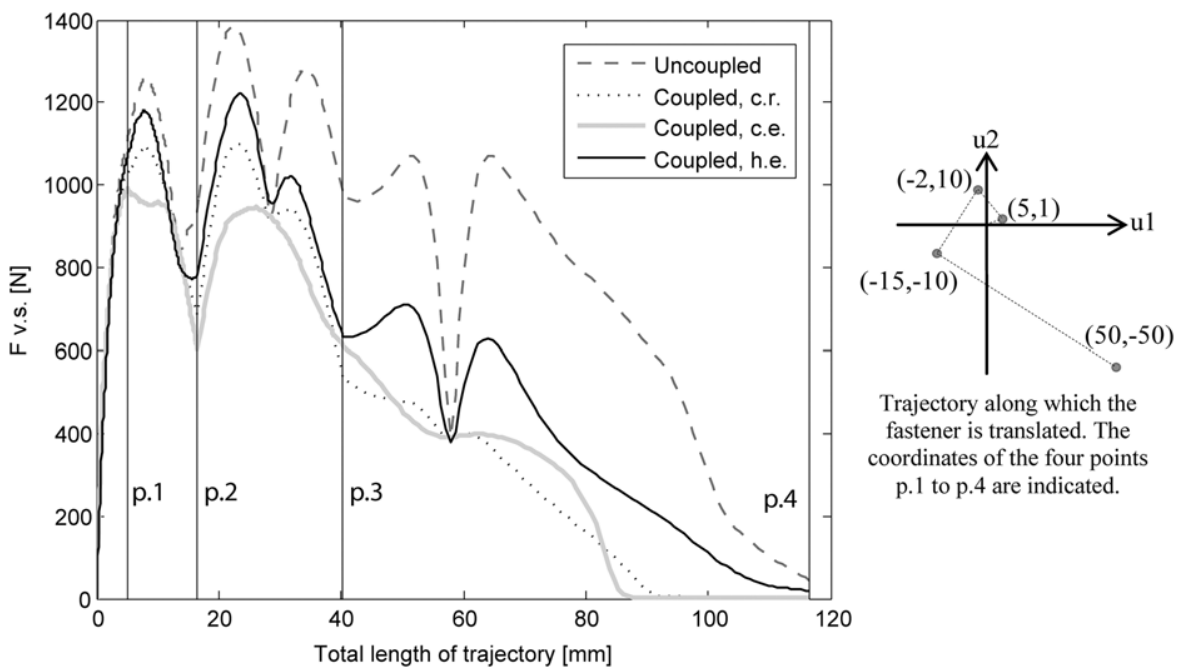

Fig. 10. Vector sum of the total force acting on the joint when deformed along the predefined trajectory.

\subsection{Modelling and analysis of a shear wall}

In order to investigate the significance of the choice of connection model on the wall level the four candidates presented were used in a finite element analysis of a shear wall in the commercial finite element software ABAQUS (version 6.8). The framing, sawn out of Norway spruce to the dimensions $45 \times$ $120 \mathrm{~mm}$, was represented with quadratic shear flexible beam elements denoted "B22". For these elements the modulus of elasticity was set to $12 \mathrm{GPa}$ and the shear modulus was $0.75 \mathrm{GPa}$. The sheathing was represented by elements denoted "CPS4R", a bilinear four node plane stress element that uses reduced integration. The modulus of elasticity was set to $6 \mathrm{GPa}$ and the shear modulus to $2.3 \mathrm{GPa}$.

The fasteners connecting the sheathing to the framing were modelled to be at a distance of $100 \mathrm{~mm}$ along the periphery of the sheathing and at $200 \mathrm{~mm}$ distance along the centre stud. For all connections between studs and rails a special stud-to-rail joint was used, which is further described below.

When analysing not only a single joint, but a complete shear wall segment, the joints fastening the timber members to each other are also vital for the performance. In many suggested models the framing members have been assumed to be linked to each other by frictionless hinges (see e.g. [14]), but in this paper stiffness is introduced in two orthogonal directions in each such joint. These joints may be designed in different ways, ranging from very slender nails fastened through the rail into the vertical stud, to efficient brackets fastened to the rail and stud respectively by means of anchor nails. In the current study these joints are given uncoupled properties according to Fig. 11 in the horizontal and vertical directions respectively. These estimates are based on experimental tests performed, see [15].

\subsubsection{Wall geometry and loading}

All loading was applied to the shear wall using displacement control in order to make it possible to trace any possible post peakload behaviour. The bottom rail was assumed to interact fully with the substrate. In the evaluation only the horizontal component of the total load is shown as a function of the horizontal displacement of the top right part of the rail. Two different shear wall geometries were analysed. The first consisted of a single rectangular wall element as shown in Fig. 12. The loading was applied in the node furthermost to the left in the top rail and was directed towards the node furthermost to the right of the bottom rail. In the second, for which the load was applied in the same way as in the first one, a lower part was attached to the right giving the shear wall geometry shown in Fig. 13. The diagonal load case approximately corresponds to the case when the leading stud is prevented from uplifting, since one of the components of the diagonal load acts vertically in compression of the leading stud. It is therefore called fully anchored, see e.g. [16], who used the fully anchored model in their analytical hand calculation models. When analysing a diagonally loaded shear wall it is often assumed that the joints are loaded parallel to the timber member, see for instance Eurocode 5 [17] and the lower bound method presented by Källsner and Lam [14] and Källsner and Girhammar [16]. This approximation could often hold, at least for connectors not too close to the corners.

\subsubsection{Results}

The fact that a joint at a distance from a corner is loaded mostly parallel to the member in the current load case implies that the difference should not be very large between the different sheathing-to-framing models. The deformed state is depicted for maximum load at the bottom right in Fig. 12, and it is obvious that close to the corners, the joints are far from being loaded parallel to the timber member. This is also verified by the load-displacement relations shown in Fig. 12. The peak values are 13.9, 13.2, 12.7 and $13.8 \mathrm{kN}$ for the u.c., the c.r., the c.e. and the h.e. models respectively. This implies that the difference is at most around $10 \%$ for the four models for this load case. As expected, the uncoupled model gives the highest peak value since it overestimates the strength of the individual sheathing-to-framing joints when loaded in any direction different from $u 1$ or $u 2$. Comparing the three coupled models, the one with a hyper-elastic model gives the highest strength values. This is due to the fact that higher capacities are obtained also on the single joint level when loaded in an angle with respect to the timber member, see Fig. 9.

The load case presented in the previous section represents the case often used for verification of various models. This is mainly due to its relative simplicity and the fact that it may be experimentally verified without too much effort spent. In reality, however, the geometry is normally much more complex. Only by adding a $900 \mathrm{~mm}$ high part to the wall, representing e.g. the part under a window, the geometry is more complex even though no contact is assumed between the two adjacent sheets. If such a wall is loaded diagonally, in the same way as presented above using the different models of the sheathing-to-framing connections, the resulting load-displacement relations will take the form presented in Fig. 13. In the lower right corner of the figure the deformed state is shown. In this case the direction of the joint displacement is 

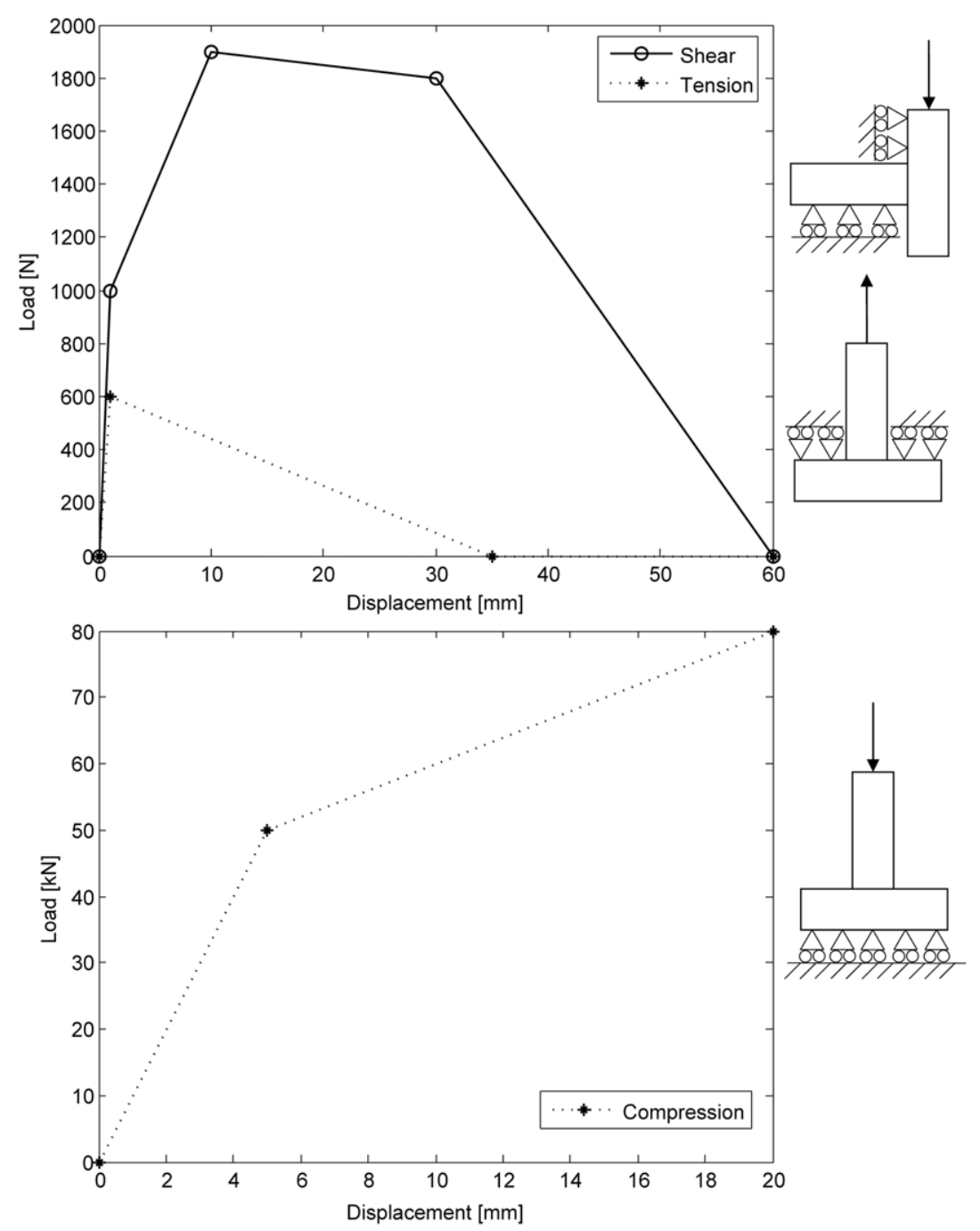

Fig. 11. Properties used in the stud-to-rail joints in horizontal direction (shear) and in vertical direction (tension and compression).

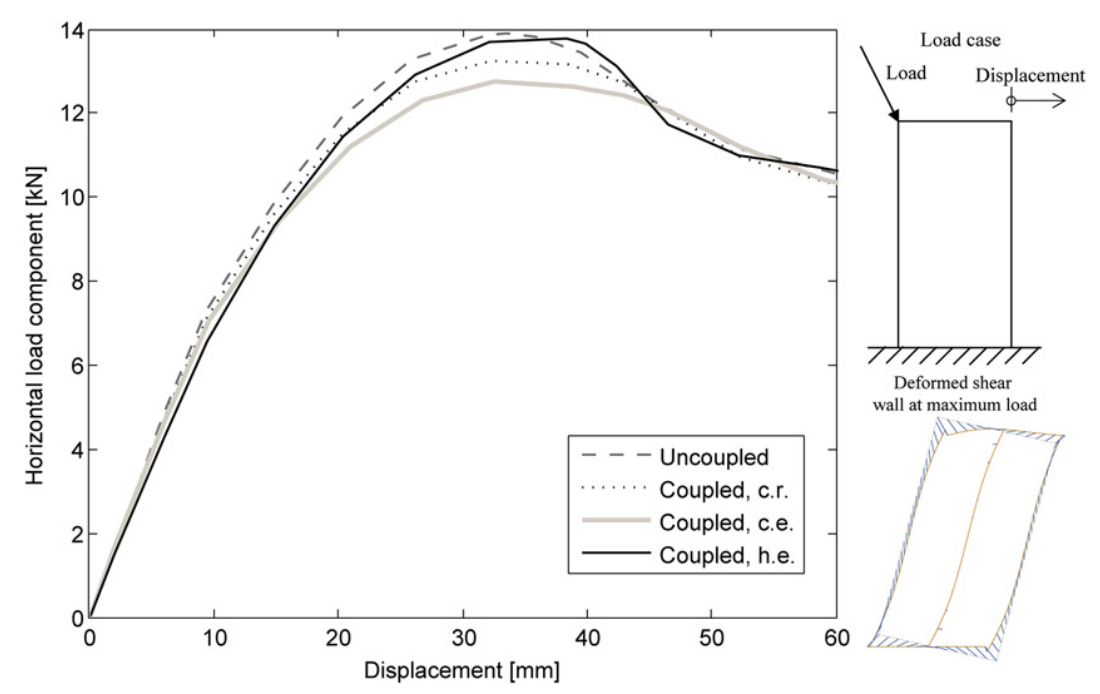

Fig. 12. Load-displacement relation for a diagonally loaded shear wall using the four different sheathing-to-framing joint assumptions: uncoupled, c.r., c.e. and h.e.

not parallel to the framework, especially not close to the $900 \mathrm{~mm}$ high part of the wall. As shown here the difference in peak load, depending on what sheathing-to-framing connection is used, is around $2 \mathrm{kN}$, corresponding to around 13\%. Again it is the uncoupled model that governs the highest load capacity while the two coupled models c.r. and c.e. give almost the same ultimate capacity. The hyper-elastic model gives a load capacity almost as high as the uncoupled model does. 

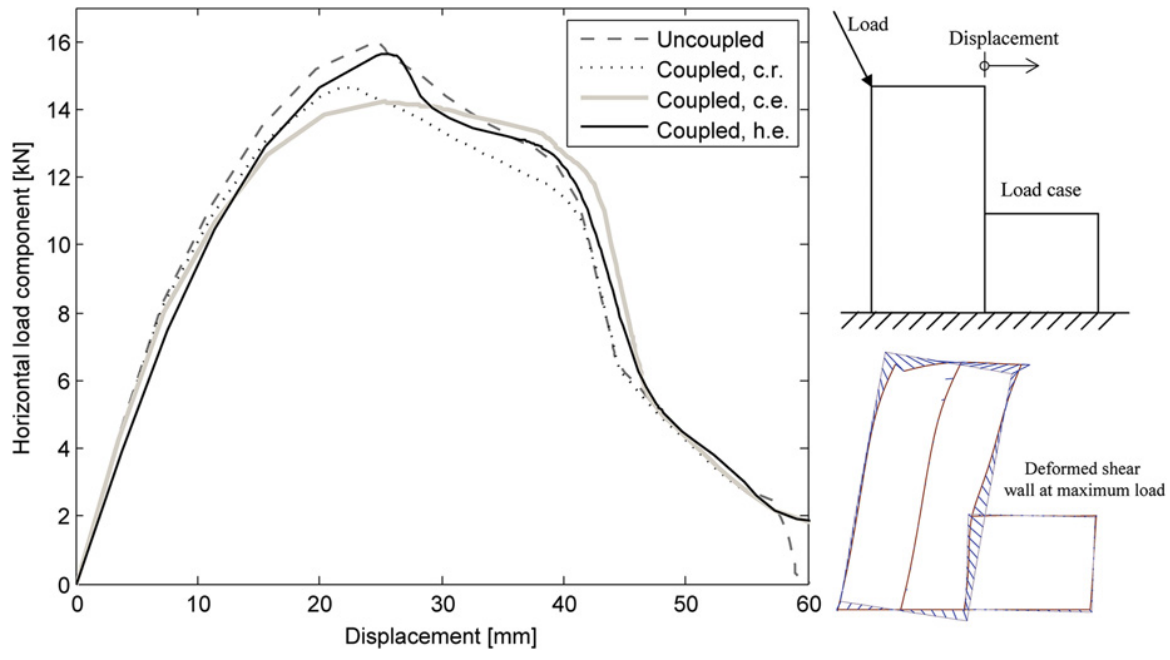

Fig. 13. Load-displacement relation for a diagonally loaded shear wall connected to a small shear wall. The response is shown for four different joint assumptions: uncoupled, c.r., c.e. and h.e.

\section{Conclusions}

Four different spring pair models, one uncoupled and three coupled, were presented and compared by means of numerical simulations. The models were analysed at the level of a single fastener, and included in models of shear walls. At the single fastener level, differences between models were illustrated by calculating the force-displacement response. On a shear wall level the differences were seen in the global load-displacement response, although the differences, as expected, were not as large as on a single fastener level. Nevertheless, differences larger than $10 \%$ in strength were shown to occur, indicating the importance of making a well-founded choice regarding the joint modelling. It is not always obvious which sheathing-to-framing model to use when analyzing timber based shear walls. The reason for this may be, for instance, the convenience in choosing the uncoupled model. The reason may also be that a path-independent, coupled model is attractive. Using such a model the order in which the external loads are applied has no influence on the calculated structural response. The following conclusions may be drawn from the present study:

- In a spring pair model consideration can be taken of the different behaviour in the parallel and perpendicular direction to a timber member. Different relations may be given in each of the two directions as compared to the commonly used single spring model which uses one load-displacement characteristic only.

- Spring pair models may be coupled or uncoupled. The uncoupled spring pair model generally overestimates the capacity of each sheathing-to-framing joint and should be used with caution. A coupled spring pair model is preferable, but must, of course, be calibrated by means of experimental tests performed for other directions than those parallel and perpendicular to the grain of the wooden members.

- The difference between the coupled models discussed in this study is limited when comparing results from simulations. Still the model with quadratic decrease and a rectangular outer bound (the c.r. model) is questionable as the displacement capacity is larger in mixed mode loading. This indicates that the model overestimates the capacity in such directions for large displacements.

- The coupled model with elliptic outer bound (the c.e. model) is a convenient way of defining the behaviour of monotonically loaded single joints. The model should enable realistic simulations considering a relatively complex joint stiffness relation without being too costly in terms of computer resources.
- The models investigated here have not been calibrated in terms of the coupling effect. They can however, be adjusted to fit a wide range of experimental data. For example, the c.e. model can be adjusted by different choices regarding the two parameters entering (7) and the choice of the scaling function (9).

- The only one of the models that is path-independent is the hyper-elastic model. Due to this fact it is attractive, although it probably overestimates the strength when loaded in an angle with respect to one of the two main directions.

\section{References}

[1] Gupta AK, Kuo GP. Modeling of a wood-framed house. J Struct Eng, ASCE 1987; 113(2):260-78.

[2] Dolan JD, Madsen B. Monotonic and cyclic nail connection tests. Can J Civ Eng 1992;19:97-104

[3] Foschi RO. Load-slip characteristic of nails. Wood Science 1974;1:69-76.

[4] Foschi RO. Modeling the hysteretic response of mechanical connections for wood structures. In: World conference on timber engineering. Vancouver: University of British Columbia; 2000.

[5] Xu J, Dolan JD. Development of nailed wood joint element in ABAQUS. J Struct Eng ASCE 2009;135(8):968-76.

[6] He M, Lam F, Foschi RO. Modeling three-dimensional timber light-frame buildings. J Struct Eng, ASCE 2001;127(8):901-13.

[7] Collins M, Kasal B, Paevere P, Foliente GC. Three-dimensional model of light frame wood buildings. I: model description. J Struct Eng 2005;131(4):676-84.

[8] Judd JP, Fonseca FS. Analytical model for sheathing-to-framing connections in wood shear walls and diaphragms. J Struct Eng, ASCE 2005;131(2):345-52.

[9] Folz B, Filiatrault AF. Cyclic analysis of wood shear walls. J Struct Eng, ASCE 2001; 127(4):433-41

[10] White MW, Dolan JD. Nonlinear shear wall analysis. J Struct Eng, ASCE 1995; 121(11):1629-35.

[11] Andreasson S. Three-dimensional interaction in stabilisation of multi-storey timber frame building systems, in division of structural engineering. Division of Structural Engineering. Lund (Sweden): Lund university; 2000.

[12] Girhammar UA, Bovim NI, Källsner B. Characteristics of sheathing-to-timber joints in wood shear walls. In: World conference on timber engineering. Lahti (Finland): 2004.

[13] Vessby J. Shear walls for multi-storey timber buildings. Thesis for the degree of licentiate of engineering. School of Technology and Design. Växjö (Sweden): 2008.

[14] Källsner B, Lam F. Diaphragms and shear walls, in Holzbauwerke nach Eurocode 5-STEP 3. Düsseldorf (Germany): 1995. p. 15/1-15/19.

[15] Palm S. Provning av knutpunktsförband i skiv-regelväggar. Report 2002:3. Faculty of Science and Technology, Department of TFE-Civil Engineering. Umeå (Sweden): Umeå University; 2003 [in Swedish].

[16] Källsner B, Girhammar UA. Plastic models for analysis of fully anchored lightframe timber shear walls. Eng Struct 2009;31(9):2171-81.

[17] European Committee for Standardization (CEN), Eurocode 5-Design of timbe structures-Part 1-1: General-Common rules and rules for buildings. prEN 1995-1-1; 2003. 